\title{
Supporting Information \\ Antibacterial Nanoparticles with Natural Photosensitizers Extracted from Spinach Leaves
}

\author{
Pavel Ludačka, ${ }^{\dagger}$ Pavel Kubát, ${ }^{\dagger *}$ Zuzana Bosáková, ${ }^{\dagger}$ and Jiř́ Mosinger ${ }^{\dagger *}$ \\ ${ }^{\dagger}$ Faculty of Science, Charles University, 2030 Hlavova, 12843 Prague 2, Czech Republic \\ $\$$ J. Heyrovský Institute of Physical Chemistry of the Czech Academy of Sciences, v.v.i., \\ Dolejškova 3, 18223 Prague 8, Czech Republic
}

\section{Content}

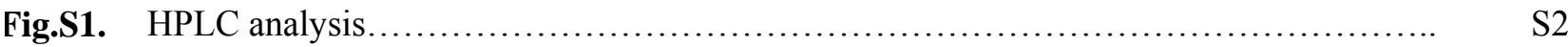

Fig.S2. Structures of chlorophyll a, $\beta$-carotene, chlorophyll $b$, and lutein $\ldots \ldots \ldots \ldots \ldots \ldots \ldots \ldots \ldots \ldots \ldots$

Fig.S3. $\quad \mathrm{O}_{2}\left({ }^{1} \Delta_{\mathrm{g}}\right)$ luminescence of absorbance matched $\mathrm{Chl} a$, ChlE (b) and $\mathrm{ZnPc}$ in THF............ S5

Fig.S4. Time resolved kinetics of $\mathrm{O}_{2}\left({ }^{1} \Delta_{\mathrm{g}}\right)$ : effect of ChlE dilution and irradiation .................. S6

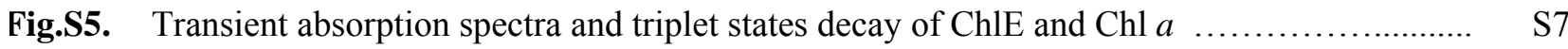

Fig.S6. Preparation scheme of sulfonated ChlE@NPs..................................................................... S8

Fig.S7. Filtration of ChlE@NPs through nanofiber membrane ................................... S8

Fig.S8. Decay of the triplet states in sulfonated ChlE@NPs .................................... S9

Fig.S9. Singlet oxygen sensitized delayed fluorescence (SODF) of NPs....................... S9

Fig.S10. Photodegradation of sulfonated and non-sulfonated ChlE@NPs ...................................... S10

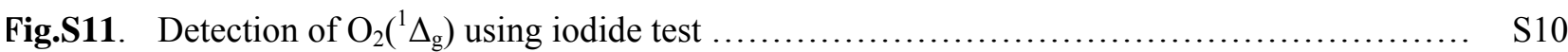

Fig.S12. Detection of $\mathrm{O}_{2}\left({ }^{1} \Delta_{\mathrm{g}}\right)$ using photobleaching of uric acid............................ S11

Fig.S13 The effect of preirradiation on the normalized kinetics of photooxidation ................ S11

Fig.S14. Emission spectrum of 500W Xe-lamp and 36W red LED grow light bulb. .............. S12

Tab.S1. Extinction coefficients of leaf pigments in THF ................................... S12

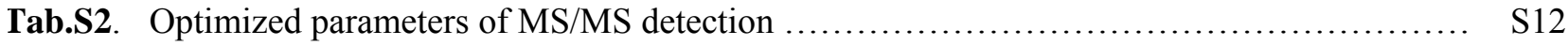

Tab.S3. Stability of the size and zeta-potential of ChlE@NPs vs time ........................... S13

Tab.S4 CFUs of E. Coli observed on agar plates after irradiation with VIS light ................. S13 

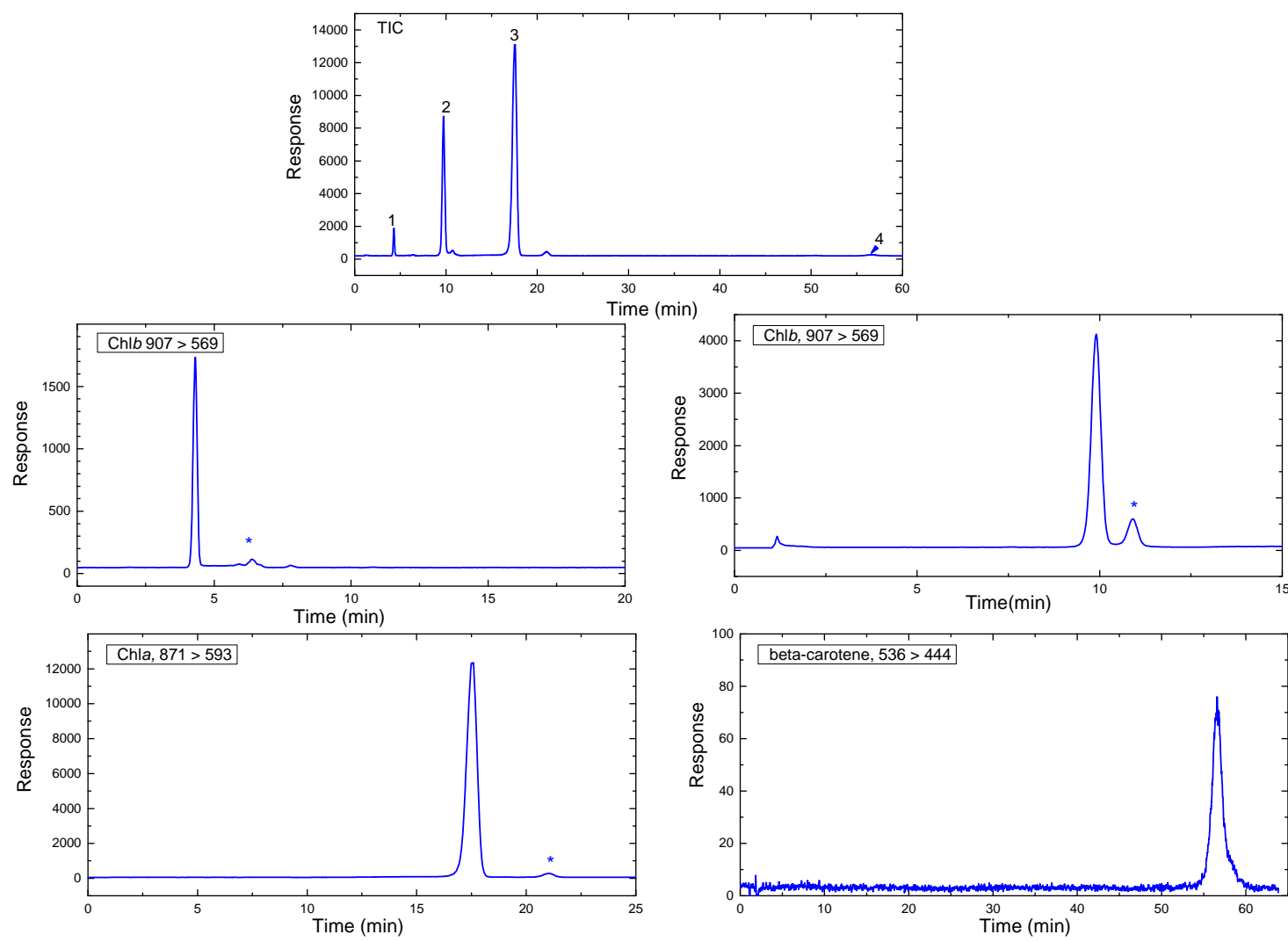

Panel A
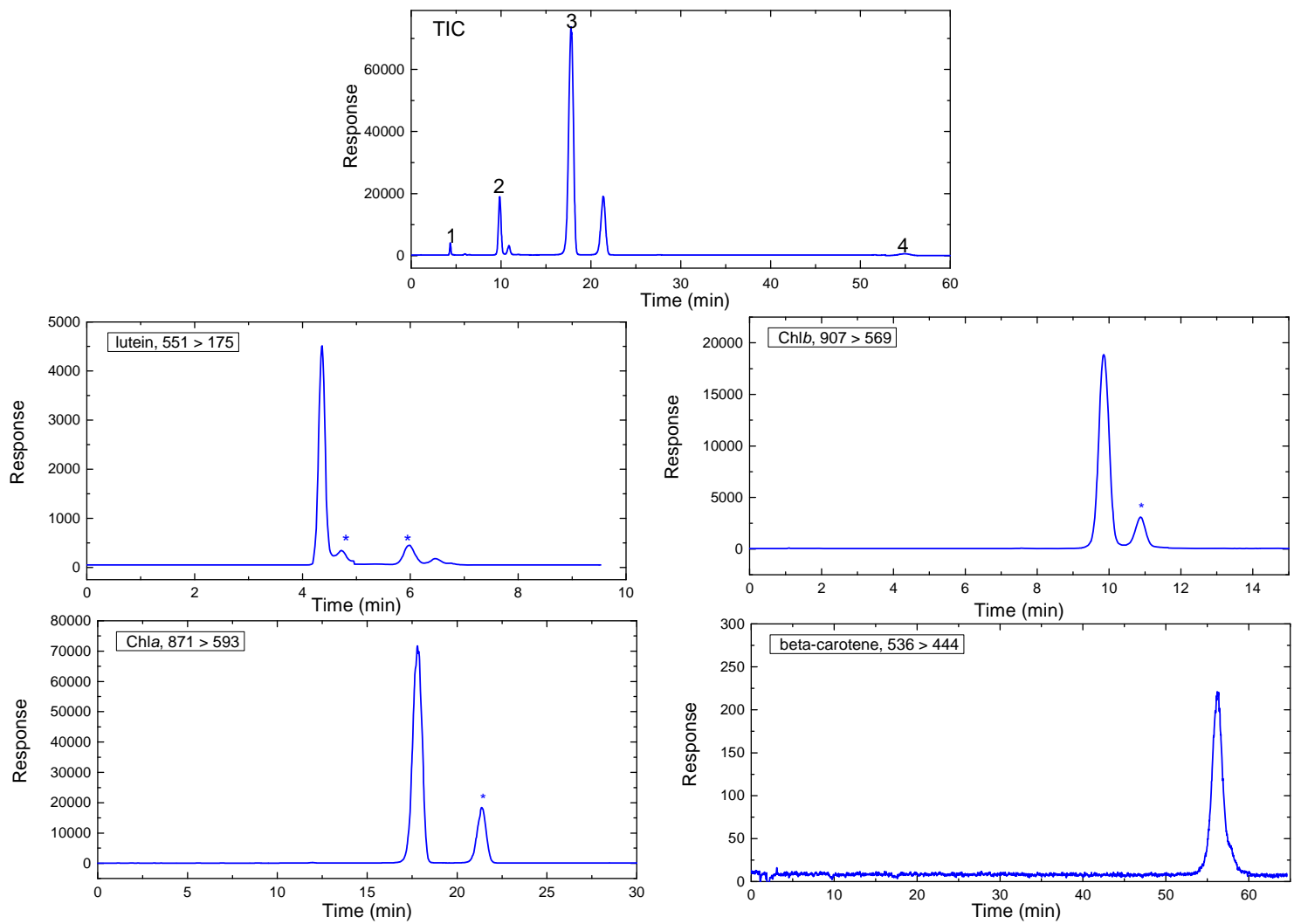

Panel B 

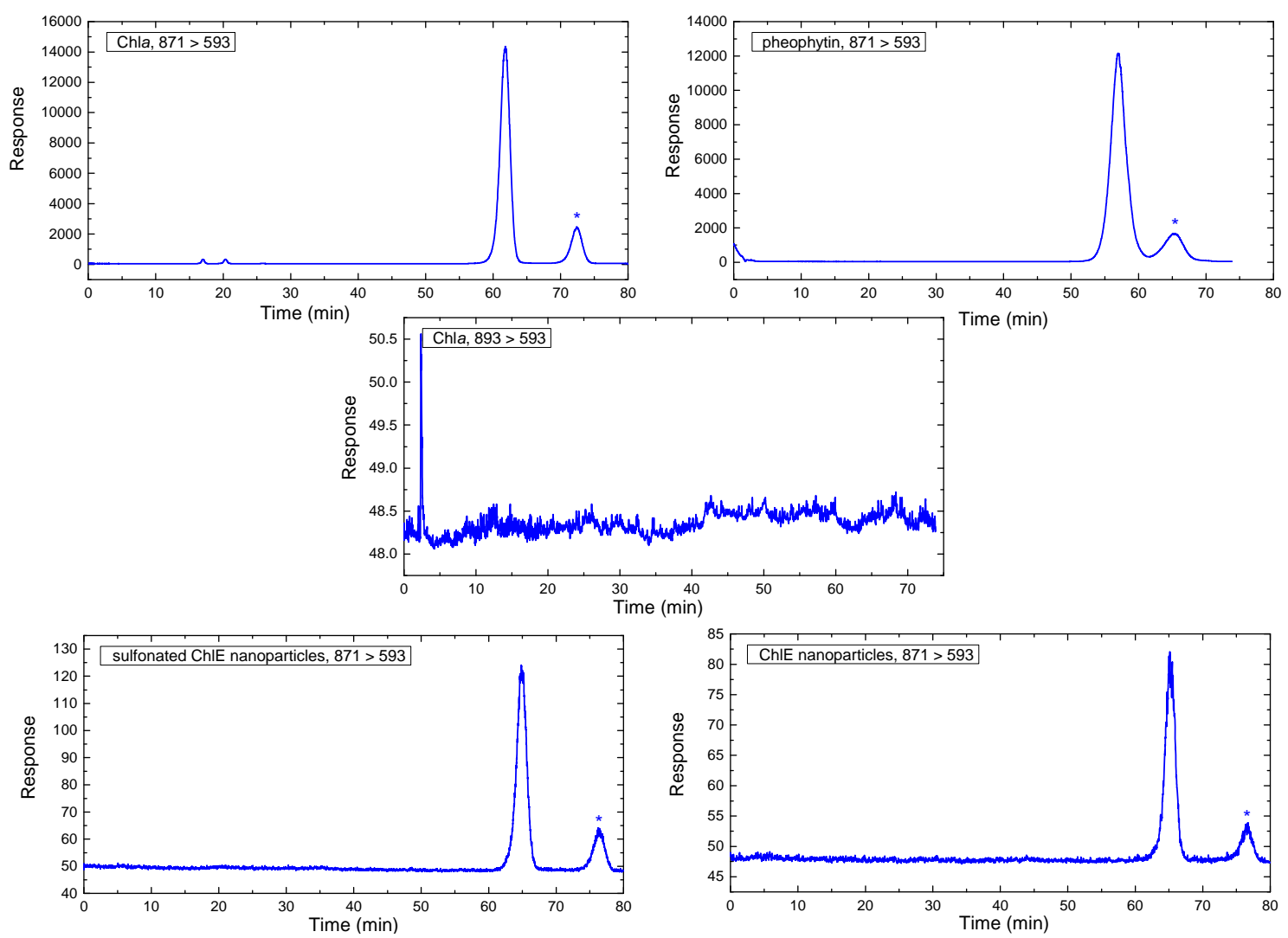

Panel C

Figure S1. Panel A: Total ion current (TIC) chromatogram of a mixture of standards $(c=$ $2.5 \mathrm{mg} / \mathrm{l})$ in MEOH/THF 9:1 $(\mathrm{v} / \mathrm{v})$ and MRM chromatograms of the individual standards, monitored at the given transitions. Lutein is assigned as 1 , chlorophyll $b(\mathrm{Chl} b)$ as 2 , chlorophyll $a(\mathrm{Chl} a)$ as 3 , and beta-carotene as 4 ; mobile phase: methanol with $0.5 \%$ formic acid. The peaks marked with an asterisk probably belong to the isomers of the corresponding chlorophylls and carotenoids ${ }^{1-3}$.

Panel B: Total ion current (TIC) chromatogram of ChlE diluted by $\mathrm{MeOH}$ and MRM chromatograms of the individual analytes in ChlE, monitored at the given transitions. Lutein is assigned as 1 , chlorophyll $b(\mathrm{Chl} b)$ as 2 , chlorophyll $a(\mathrm{Chl} b)$ as 3 and beta-carotene as 4; mobile phase: methanol with $0.5 \%$ formic acid. The peaks marked with an asterisk probably belong to the isomers of the corresponding chlorophylls and carotenoids. ${ }^{1-3}$

Panel C: To exclude or confirm the demetallization of Chla during the preparation of ChlE or the chromatographic process, the standards of Chla and pheophytin in THF were analyzed in the mobile phase with $100 \%$ methanol content with the absence of formic acid. Chla and pheophytin were monitored at transition $871 \rightarrow 593$ and Chla was additionally monitored at transition $893 \rightarrow 593$ (Chla with a centrally bound $\mathrm{Mg}$ atom). Chla and pheophytin show different retention patterns at transition $871 \rightarrow 593$ and MRM chromatogram of Chla at transition $893 \rightarrow 593$ does not contain any peak. Therefore, demetallization takes place only in the ion source of MS detection. This figure also shows MRM chromatograms of samples obtained by dissolving sulfonated ChlE@NP and non-sulfonated ChlE@NP, confirming the incorporation of Chla into NPs. Mobile phase 100\% methanol, other chromatographic and detection conditions the same as described in the main manuscript (HPLC analysis). The 
peaks marked with an asterisk probably belong to the isomers of the corresponding chlorophylls and carotenoids. ${ }^{1-3}$

\section{Note to Figure S1}

The peak at 6 min occurs in both the MRM chromatogram of the lutein standard (panel A) and the MRM chromatogram of the lutein found in the extract (panel B). The main peak eluting at $4.3 \mathrm{~min}$ and the peak eluting at $6.4 \mathrm{~min}$ show the same fragmentation pattern, i.e. transition $551 \rightarrow 175$. The mass spectrum shows the protonated molecule at $\mathrm{m} / \mathrm{z} 551$, resulting from the loss of water $[\mathrm{M}+\mathrm{H}-18]^{+}$from the polyene chain of lutein. The detailed MRM chromatogram of lutein standard and lutein in the extract contains 4 peaks, one main at $4.3 \mathrm{~min}$ and three minor between 4.9 and $6.8 \mathrm{~min}$, which are more pronounced in the extract. All four peaks show the same $\mathrm{m} / \mathrm{z}$ transition $551 \rightarrow 175$. These are believed to be lutein isomers. It is known that lutein undergoes isomerization in light. It can be assumed that the degree of isomerization of the freshly prepared standard, stored in the dark and cold, will be significantly lower than in the case of ChlE extract. A similar situation is also for Chla (17.8 and $21.4 \mathrm{~min}$ ) and $\mathrm{Chl} b$ (9.9 and $10.9 \mathrm{~min}$ ), where the minor peaks present in the MRM chromatograms of the relevant standards are smaller, those found in the extracts, especially for Chla. Also, the minor peaks show the same MRM transitions as the respective major peaks, so they are most likely their isomers. Standards for the respective isomers were not commercially available. These peaks are marked with an asterisk as isomers.

\section{REFERENCES}

(1) Zeb, A.; Nisar, P. Effects of High Temperature Frying of Spinach Leaves in Sunflower Oil on Carotenoids, Chlorophylls, and Tocopherol Composition. Front. Chem. 2017, 5.19.

(2) Li, D.; Xiao, Y.; Zhang, Z.; Liu, Ch. Analysis of (all-E)-lutein and its (Z)-isomers during illumination in model system. J. Pharm. Biomed. 2014, 100, 33.

(3) Hitoshi, M.; Tadashi, W.; Terumi, T.; Andreas, S.; Hugo, S. Epimerization of chlorophyll derivatives, Effects of the central magnesium and ring substituents on the epimerization of chlorophyll derivatives. Bull. Chem. Soc. Jpn. 1992, 65, 3080. 


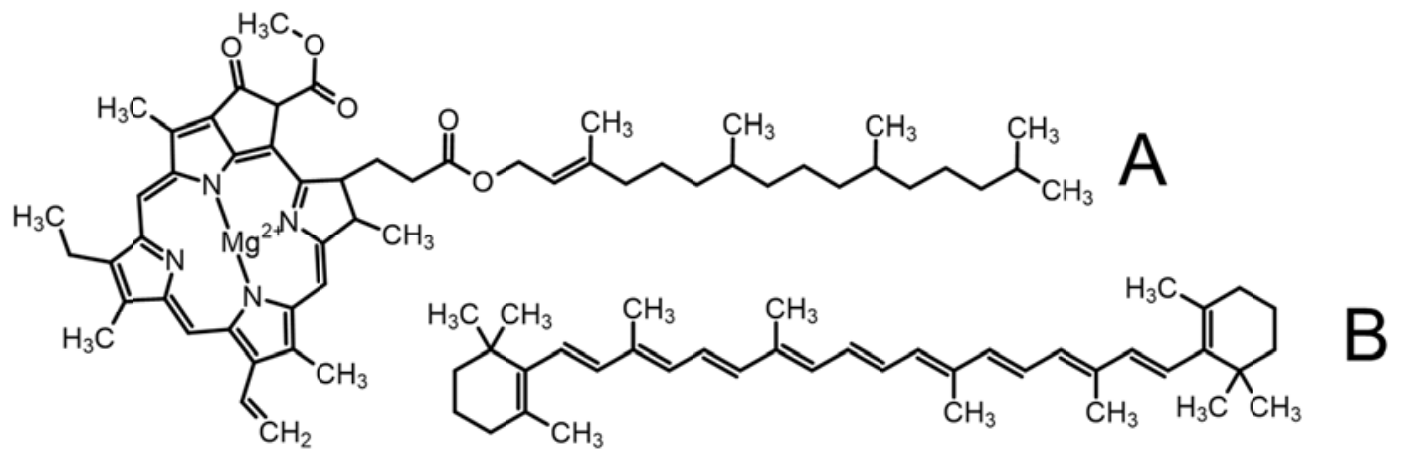

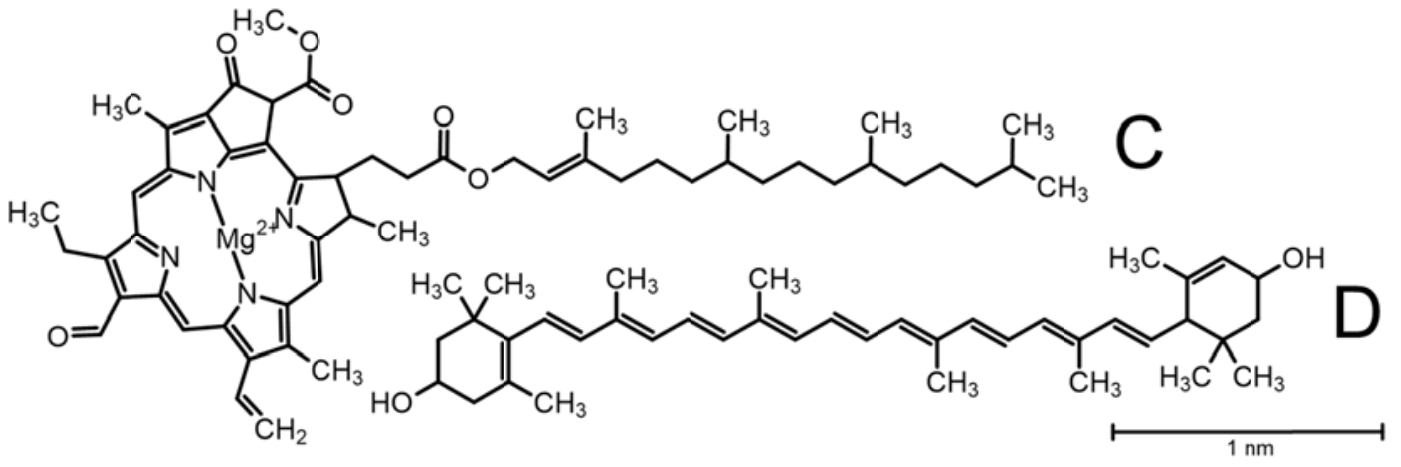

Figure S2. Structures of chlorophyll $a$ (A), $\beta$-carotene (B) chlorophyll $b(\mathrm{C})$ and lutein (D).
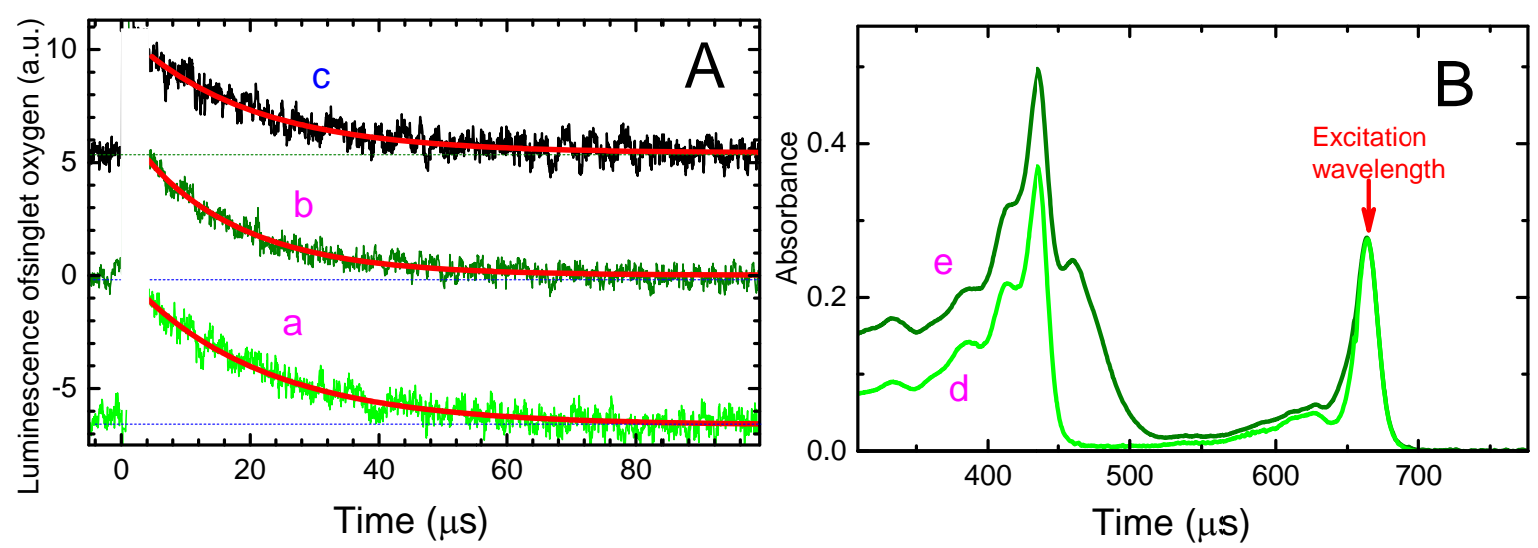

Figure S3. Panel A: Singlet oxygen luminescence for Chl $a$ (a), ChlE (b) and standard ZnPc (c) in THF. Panel B: Corresponding UV/vis spectra of Chl $a$ (d) and ChlE (e) in THF. Excitation wavelength at $664 \mathrm{~nm}$. 

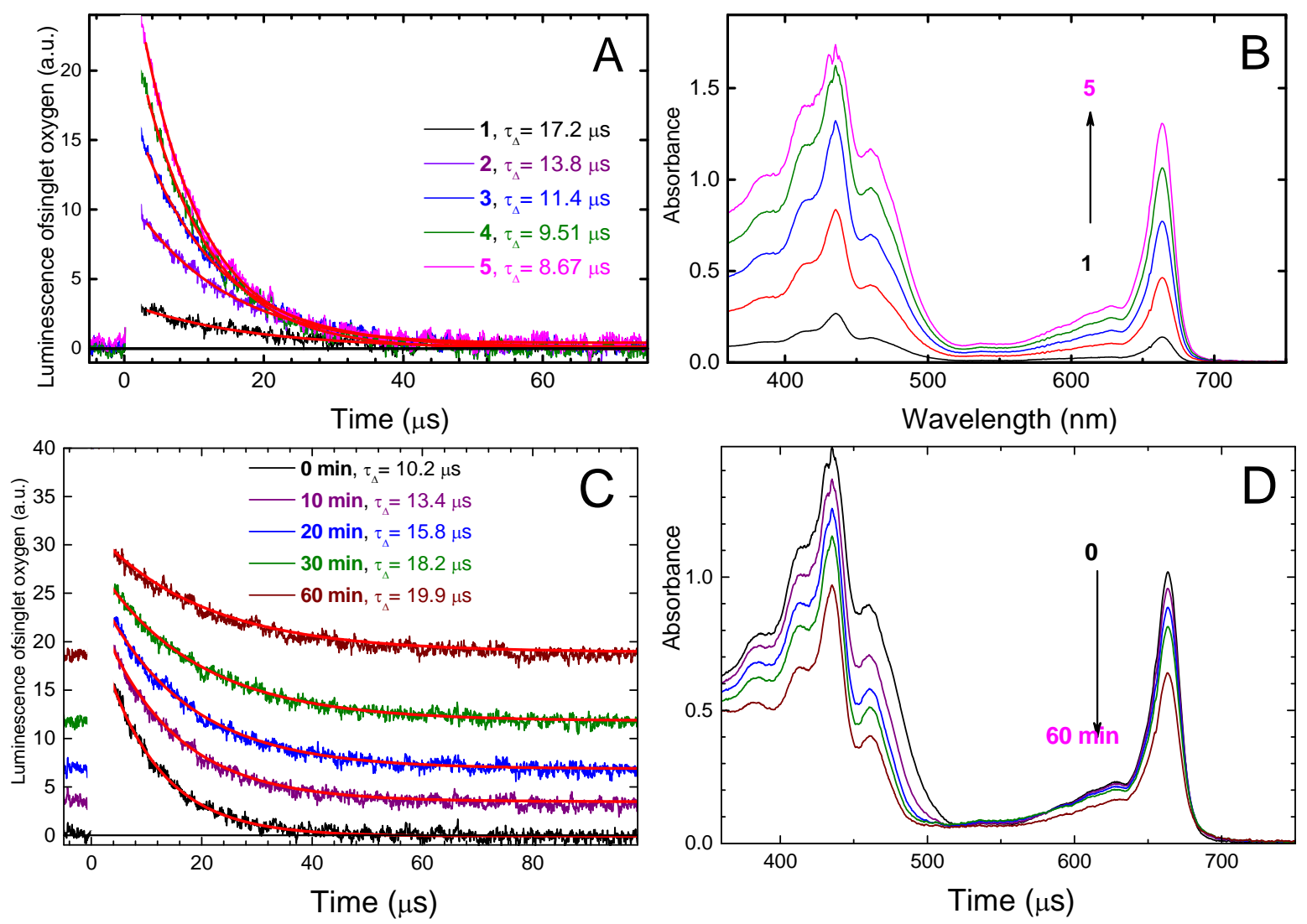

Figure S4. Time resolved kinetics of singlet oxygen after excitation of ChlE (panels A ) and preirradiated ChlE* (panels C) in THF by a $28 \mathrm{~ns}$ pulse of the red laser $(665 \mathrm{~nm}$ ) (panels A and C) with corresponding UV/vis absorption spectra (panels B and D). The red lines (panels $\mathrm{A}$ and C) are single exponential fits into experimental data. Irradiated by $200 \mathrm{~W}$ Xe lamp with long pass filter $(\lambda>400 \mathrm{~nm})$. 

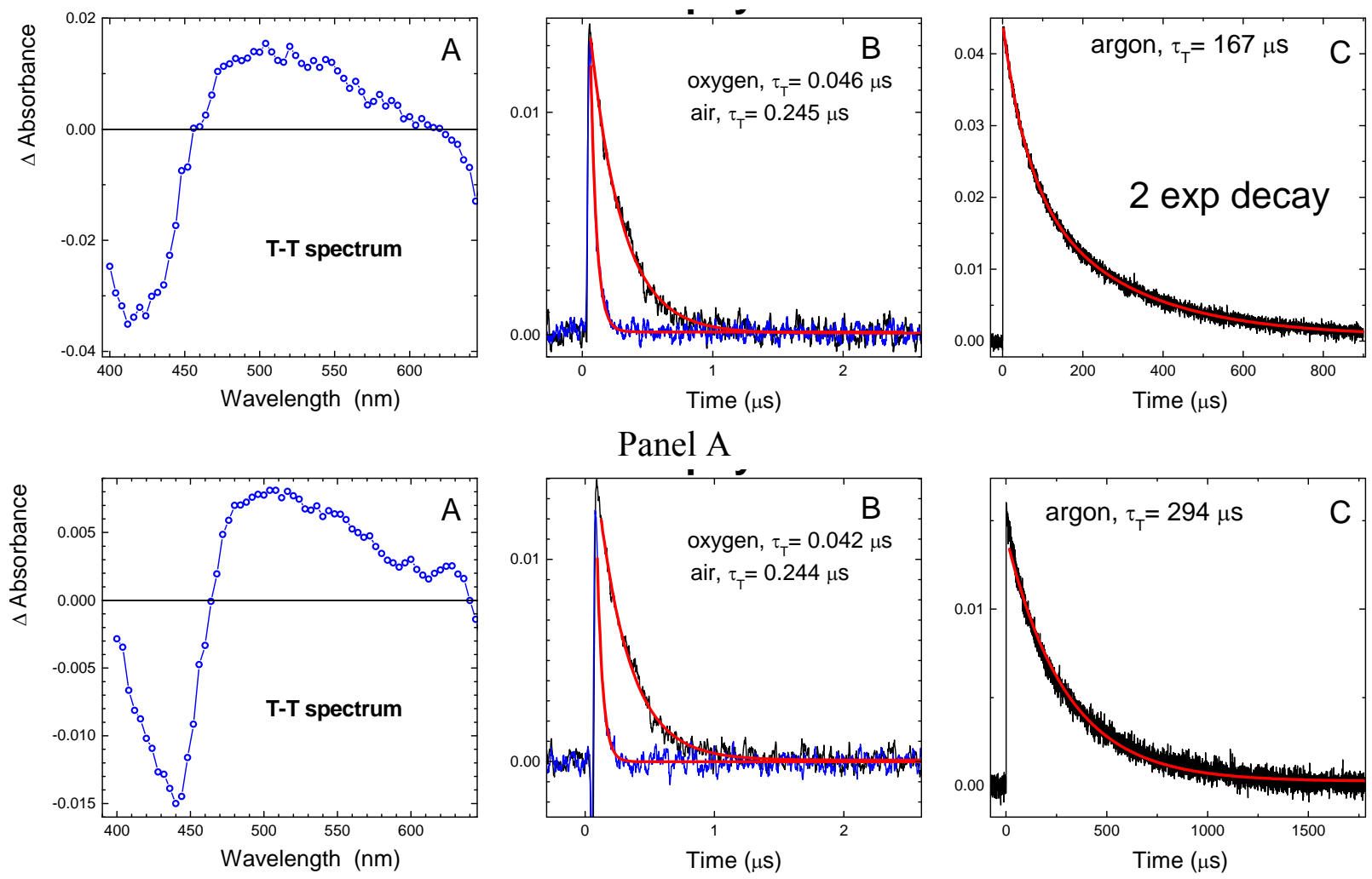

Panel B

Figure S5. Panel A: Transient absorption spectrum of ChlE in THF (panel A) after excitation of a $665 \mathrm{~nm}$ laser pulse (pulse width of $28 \mathrm{~ns}$ )and corresponding kinetic decays at $480 \mathrm{~nm}$ in oxygen-, air- (both panel B) and argon- (panel C) saturated THF. Panel B:Transient absorption spectrum of Chla (standard) in THF (panel A) after excitation of a $665 \mathrm{~nm}$ laser pulse (pulse width of $28 \mathrm{~ns}$ )and corresponding kinetic decays at $480 \mathrm{~nm}$ in oxygen-, air- (both panel B) and argon- (panel C) saturated THF. 


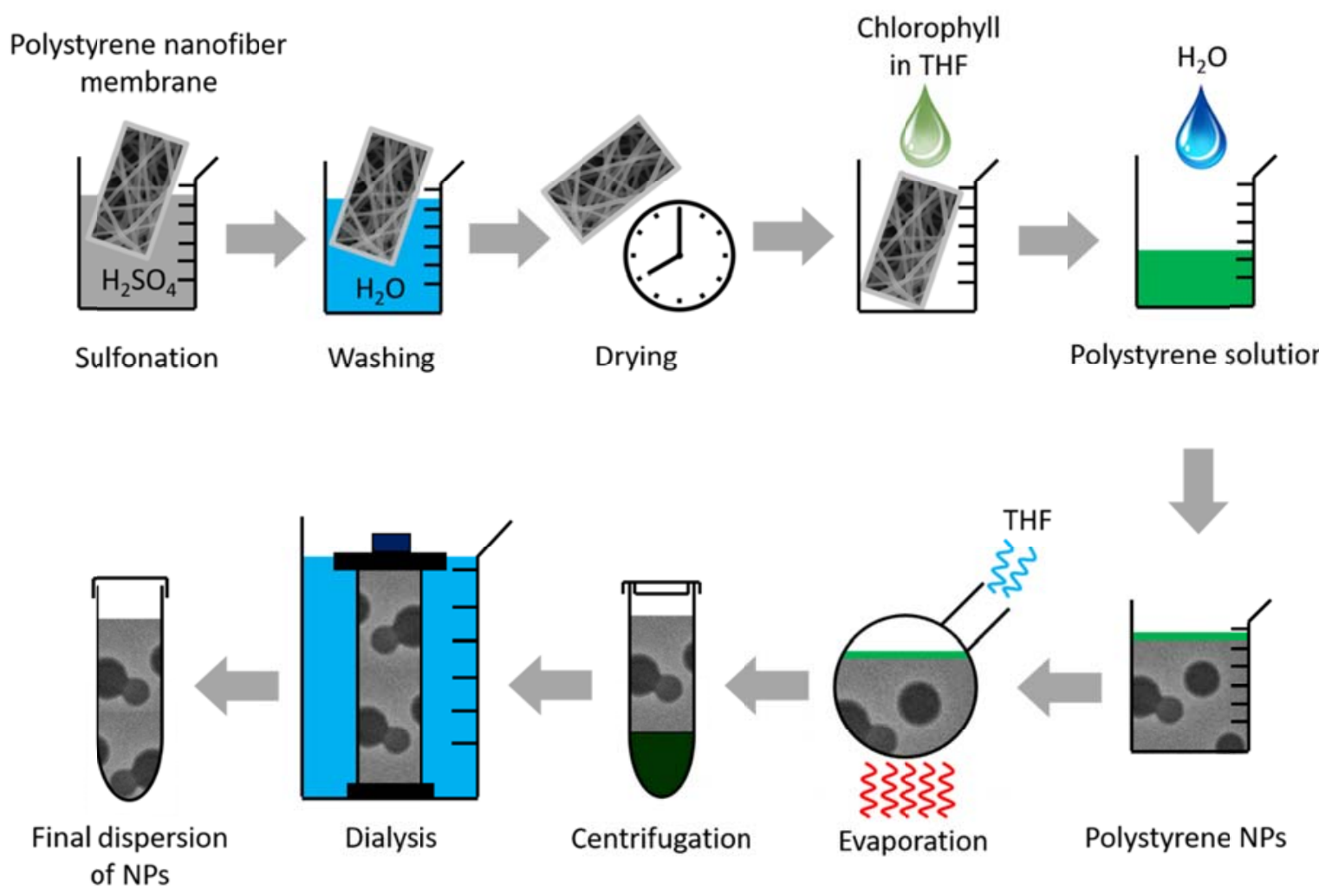

Figure S6. Preparation scheme of sulfonated ChlE@NPs.
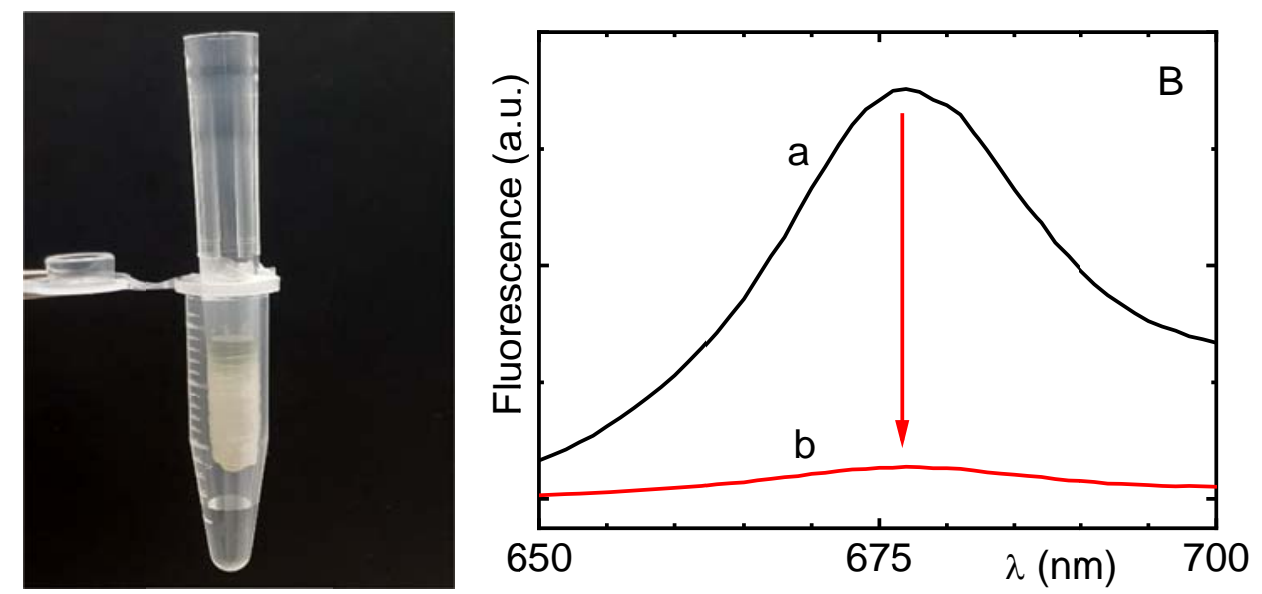

Figure S7. Panel A: Simple filtration of sulfonated ChlE@NPs through polyurethane (Tecophilic) nanofiber membrane. Panel B: The fluorescence sulfonated of ChlE@NPs before (a) and after the first filtration (b). Excitation wavelength at $420 \mathrm{~nm}$. 


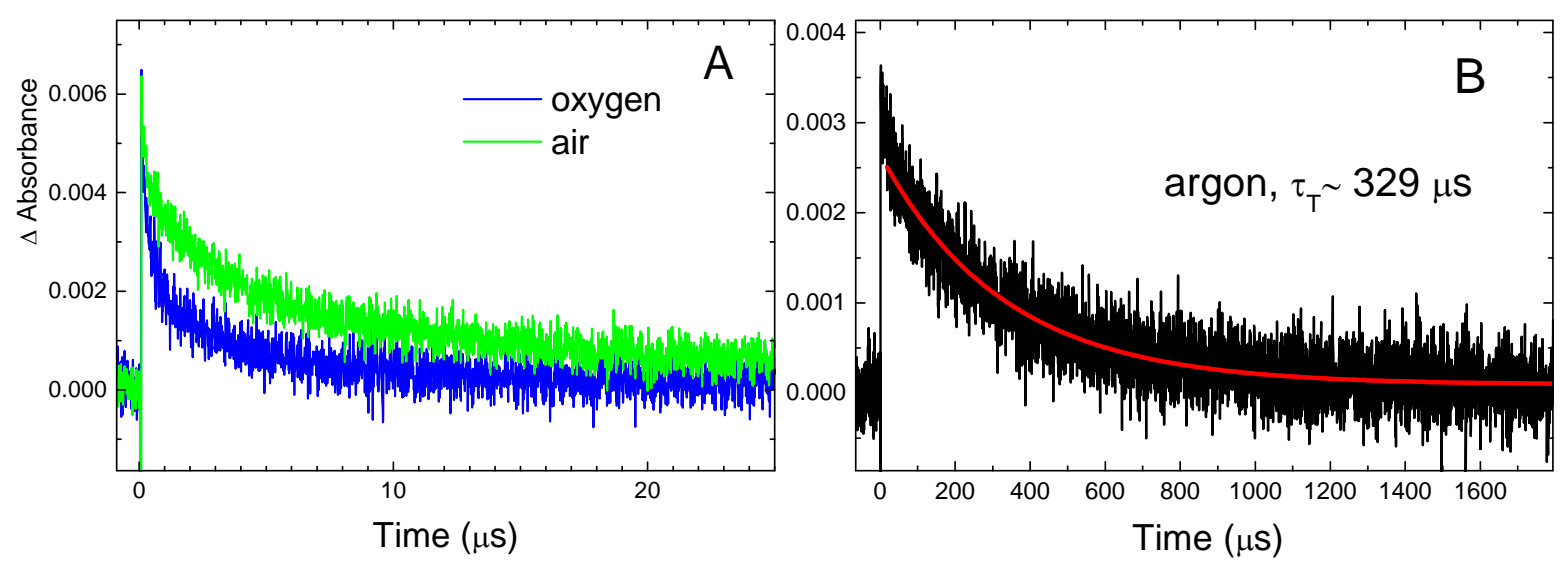

Figure S8. Kinetic decays of the triplet states of Chls in sulfonated ChlE@NPs at $520 \mathrm{~nm}$ after excitation of a $671 \mathrm{~nm}$ laser pulse in oxygen-, air- (both panel A) and argon- (panel B) saturated THF. The red line is a single exponential fit to the experimental data.

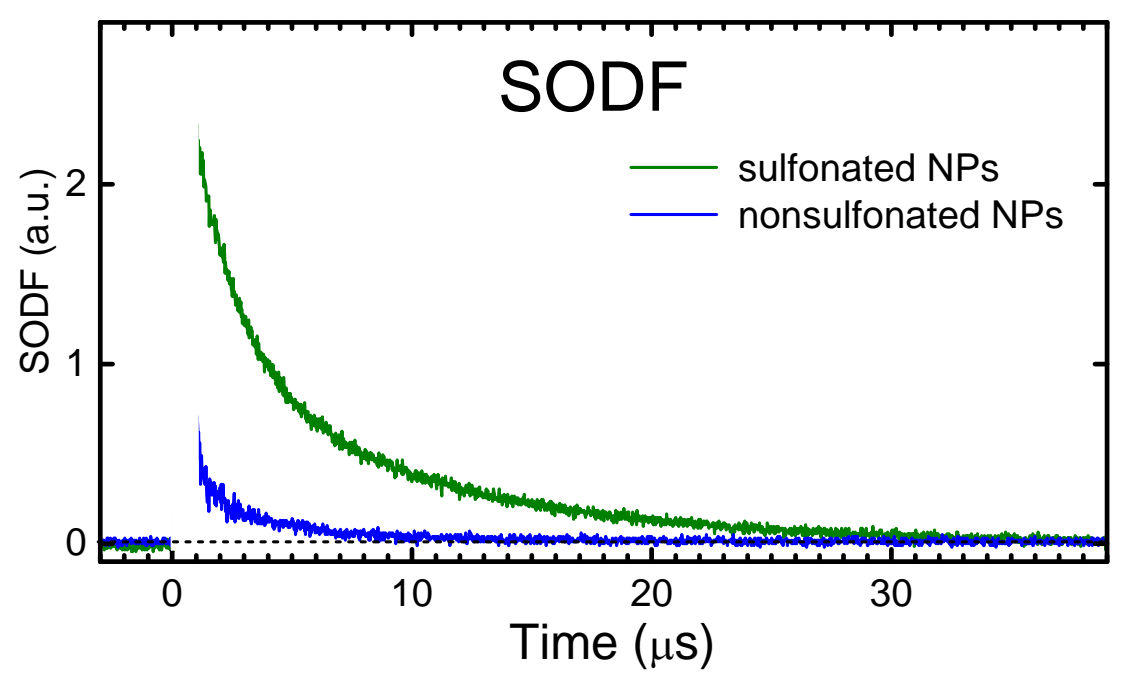

Figure S9. Comparison of SODF signals of sulfonated ChlE@NPs and non-sulfonated ChlE@NPs in aqueous dispersions. 

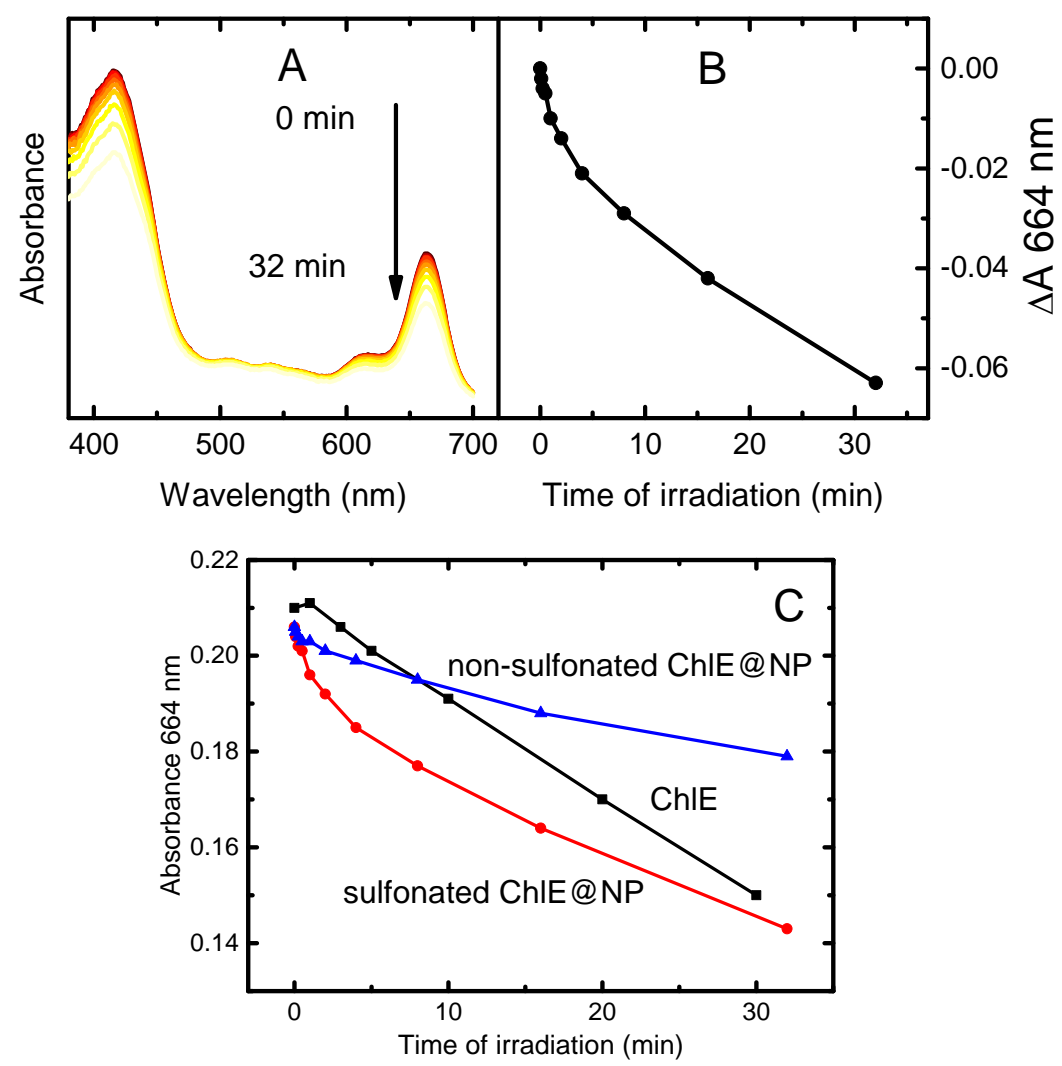

Figure S10. Panel A: VIS spectra of sulfonated ChlE@NPs $\left(6.9 \times 10^{12} \mathrm{NPs} / \mathrm{ml}\right)$ during irradiation. Panel B: Kinetics of photodegradation of Chla band at $664 \mathrm{~nm}$. Panel C: Kinetics of photodegradation of sulfonated ChlE@NPs, nonsulfonated ChlE@NPs and ChlE. 500W Xe-lamp with long pass filter $(\lambda \geq 400 \mathrm{~nm})$. Note: As non-sulfonated ChlE@NPs exhibit large light scattering, the scattering was subtracted from the original absorbance value of 0.44 .
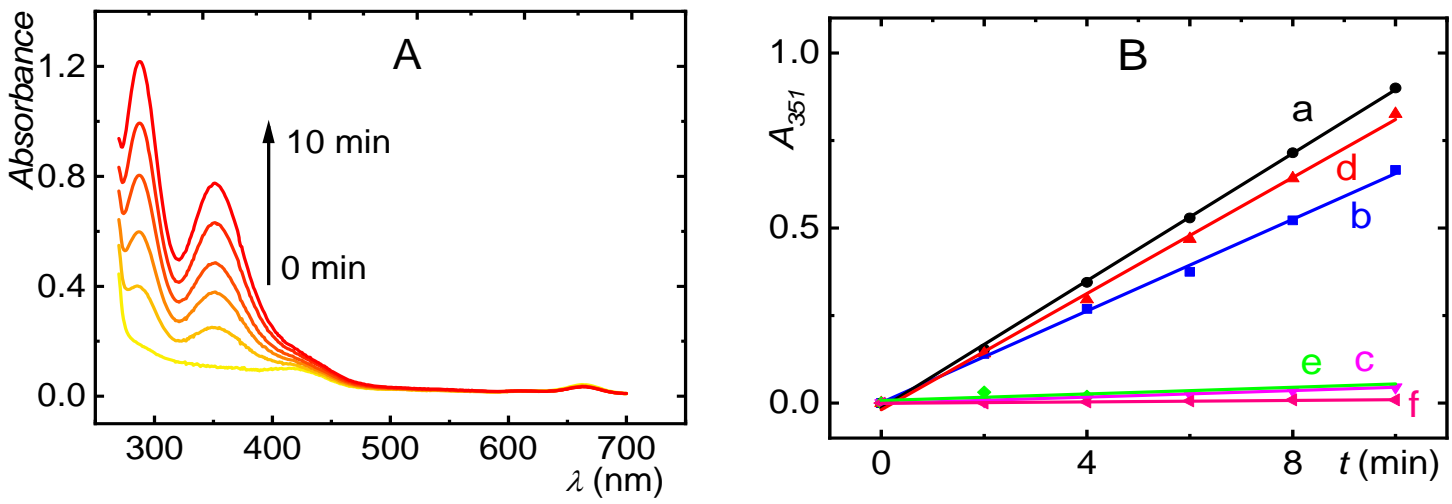

Figure S11. Photooxidation iodide test using sulfonated ChlE@NPs $\left(1.3 \times 10^{12} \mathrm{NPs} / \mathrm{ml}\right)$ : Panel A: The absorbance at $287 \mathrm{~nm}$ and $351 \mathrm{~nm}$ correspond to the amount of photogenerated $\mathrm{O}_{2}\left({ }^{1} \Delta_{\mathrm{g}}\right)$. Panel B: The kinetics of $\mathrm{I}_{3}{ }^{-}$monitored at $351 \mathrm{~nm}$ during irradiation of sulfonated ChlE@NPs, under specific conditions: dispersion saturated by $\mathrm{O}_{2}$ (a) air (b) $\mathrm{N}_{2}$ (c), airsaturated dispersion with $50 \% \mathrm{D}_{2} \mathrm{O}(\mathrm{d})$, air-saturated dispersion with $0.9 \mathrm{M} \mathrm{NaN}_{3}$ (e), and detection solution without NPs (f). Irradiation source: 500W Xe lamp with long pass filter $(\lambda \geq 400 \mathrm{~nm})$. 

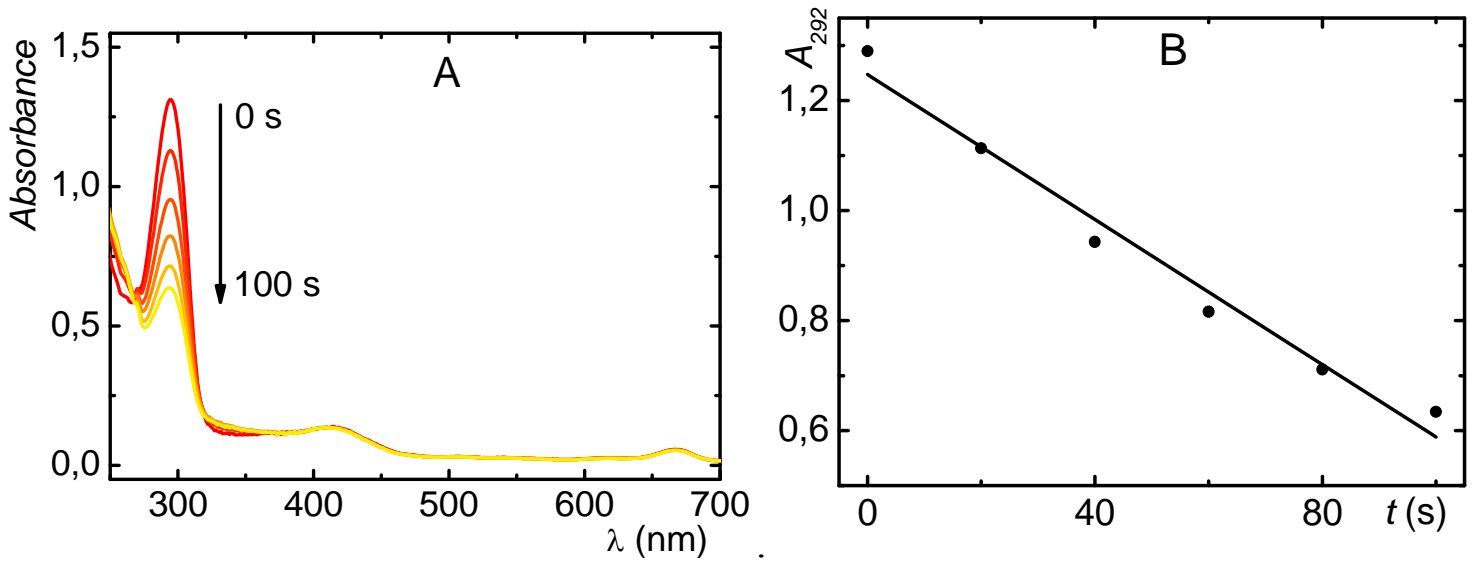

Figure S12. Detection of $\mathrm{O}_{2}\left({ }^{1} \Delta_{\mathrm{g}}\right)$ using photobleaching of uric acid. Panel A: UV/VIS spectra of $10^{-4} \mathrm{M}$ uric acid with dispersion sulfonated ChlE@NPs $\left(1.3 \times 10^{12} \mathrm{NPs} / \mathrm{ml}\right)$ at different time after irradiation. Panel B: Kinetics of photobleaching of uric acids. Irradiation source: $36 \mathrm{~W}$ red LED grow light bulb ( $\lambda=662 \mathrm{~nm})$.

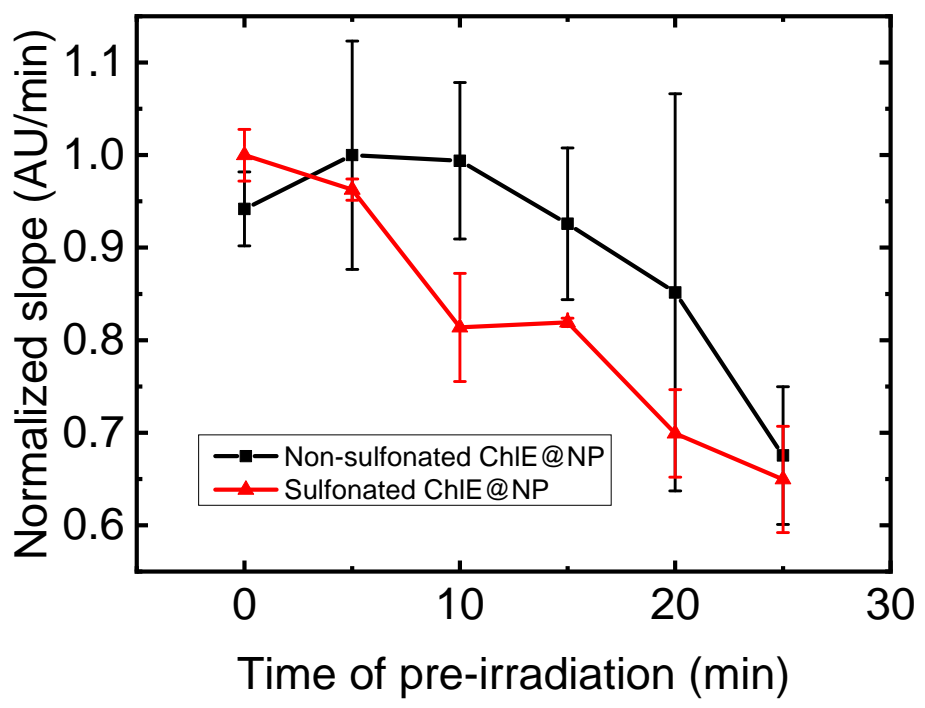

Figure S13. The effect of preirradiation on the normalized kinetics of photooxidation (d $A_{351} / \mathrm{dt}$ ) of sulfonated ChlE@NPs and nonsulfonated ChlE@NPs. 

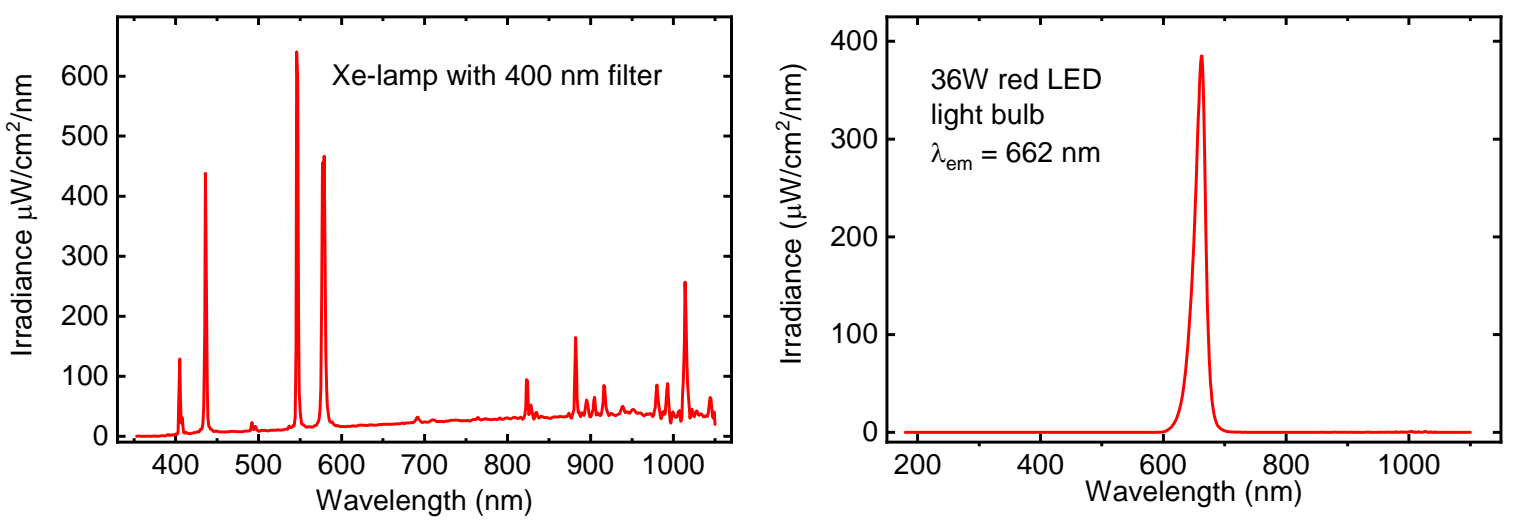

Figure S14. Emission spectrum of 500W Xe-lamp (left) and 36W red LED grow light bulb (right).

Table S1. Extinction coefficients of leaf pigments in THF calculated from Lambert - Beer equation using Sigma Aldrich standards

\begin{tabular}{lrr}
\hline \multicolumn{1}{c}{ Pigment } & $\lambda_{\max }$ & $\varepsilon\left(1 \mathrm{~mol}^{-1} \mathrm{~cm}^{-1}\right)$ \\
\hline Lutein & 453 & 106200 \\
$\beta$-Carotene & 459 & 9700 \\
Chlorophyll $b$ & 649 & 65600 \\
Pheophorbide $\left.a^{\text {a }}\right)$ & 654 & 33100 \\
Chlorophyll $a$ & 664 & 92900 \\
Pheophytin $\left.a^{\text {a }}\right)$ & 668 & 43500 \\
\hline
\end{tabular}

a) Estimated value from analytical standard chlorophyll $a$ using $\mathrm{H}_{2} \mathrm{SO}_{4}$ decomposition.

Table S2. Optimized parameters of MS/MS detection.

\begin{tabular}{lllll}
\hline Compound & $\begin{array}{l}\text { Precursor } \\
\text { Ion }\end{array}$ & Product Ion & $\begin{array}{l}\text { Fragmentor } \\
(\mathrm{V})\end{array}$ & $\begin{array}{l}\text { Collision } \\
\text { Energy }(\mathrm{V})\end{array}$ \\
\hline Lutein & 551 & 175 & 135 & 15 \\
$\beta$-Carotene & 536 & 444 & 135 & 20 \\
Chlorophyll $a$ & 871 & 593 & 135 & 45 \\
Chlorophyll $b$ & 907 & 569 & 135 & 35 \\
\hline
\end{tabular}


Table S3. Time stability of size and zeta-potential of sulfonated ChlE@NPs.

\begin{tabular}{ccc}
\hline Time (days) & Size $(\mathrm{nm})$ & Zeta potential $(\mathrm{mV})$ \\
\hline 0 & 61 & -26 \\
9 & 62 & -22 \\
13 & 59 & -27 \\
63 & 61 & -29 \\
\hline
\end{tabular}

Table S4. The number of the CFUs of Escherichia coli observed on agar plates after irradiation with visible light or stored in the dark. The average number of three independent experiments, irradiation source: $36 \mathrm{~W}$ red LED bulb.

\begin{tabular}{ccccccc}
\hline \multirow{2}{*}{$\begin{array}{c}\text { Time } \\
(\mathrm{min})\end{array}$} & \multirow{2}{*}{ E.Coli } & $\begin{array}{c}\text { E.Coli }+ \\
\text { ChlE@NPs }\end{array}$ & & E.Coli & $\begin{array}{c}\text { E.Coli }+ \\
\text { @NPs }\end{array}$ & $\begin{array}{c}\text { E.Coli }+ \\
\text { ChlE@NPs }\end{array}$ \\
\hline 0 & 2422 & 2472 & & 1684 & 2195 & 2171 \\
10 & 1984 & 2009 & 1940 & 2497 & 1388 \\
20 & 2318 & 2347 & 2182 & 2141 & 0203 \\
\hline
\end{tabular}

